\title{
The Race Model Inequality: Interpreting a Geometric Measure of the Amount of Violation
}

\author{
Hans Colonius \\ Oldenburg University
}

\author{
Adele Diederich \\ International University Bremen
}

\begin{abstract}
An inequality by J. O. Miller (1982) has become the standard tool to test the race model for redundant signals reaction times (RTs), as an alternative to a neural summation mechanism. It stipulates that the RT distribution function to redundant stimuli is never larger than the sum of the distribution functions for 2 single stimuli. When many different experimental conditions are to be compared, a numerical index of violation is very desirable. Widespread practice is to take a certain area with contours defined by the distribution functions for single and redundant stimuli. Here this area is shown to equal the difference between 2 mean RT values. This result provides an intuitive interpretation of the index and makes it amenable to simple statistical testing. An extension of this approach to 3 redundant signals is presented.
\end{abstract}

Keywords: redundant signals, race model inequality, negative dependence

In the redundant signals paradigm for simple reaction time (RT), the observer must initiate a response as quickly as possible following the detection of any stimulus onset. A typical finding is that of redundancy gain: Responses are faster, on average, when two or more signals are presented simultaneously than when a single signal appears. Since the pioneering study by Todd (1912), this redundant signals effect (RSE) has been replicated many times for both manual and saccadic RTs, and under different experimental settings, for example, comparing uni- versus multimodal stimulation (Amlôt, Walker, Driver, \& Spence, 2003; Diederich, 1995; Diederich \& Colonius, 1987; Diederich, Colonius, Bockhorst, \& Tabeling, 2003; Gielen, Schmidt, \& Van den Heuvel, 1983; Hughes, Nelson, \& Aronchick, 1998; Miller, 1982, 1986; Molholm, Ritter, Javitt, \& Foxe, 2004), single versus multiple stimuli within the same modality (e.g., Schwarz \& Ischebeck, 1994), or monocular versus binocular stimulation (Blake, Martens, \& DiGianfillipo, 1980; Westendorf \& Blake, 1988) and also for specific populations (e.g., Corballis, 1998; Marzi et al., 1996, for hemianopics; Miller, 2004, for individuals who have undergone split-brain surgery; Reuter-Lorenz, Nozawa, Gazzaniga, \& Hughes, 1995; Savazzi \& Marzi, 2004).

Raab (1962) was the first to propose a race model for simple RT such that (a) each individual stimulus elicits a detection process performed in parallel to the others and (b) the winner's time determines the observable RT. This model suggests that RSE is generated by statistical facilitation: If detection latencies are interpreted as (nonnegative) random variables, the time to detect the first of several redundant signals is faster, on average, than the detection time for any single signal. A generalization of Raab's model was recently developed in Miller and Ulrich (2003).

Testing the race model amounts to testing whether an observed RT speed-up is too large to be attributed to statistical facilitation

Hans Colonius, Department of Psychology, Oldenburg University, Oldenburg, Germany; Adele Diederich, School of Humanities and Social Sciences, International University Bremen.

Correspondence concerning this article should be addressed to Hans Colonius, Department of Psychology, University of Oldenburg, Oldenburg, Germany. E-mail: hans.colonius@uni-oldenburg.de (viz., probability summation). The race model inequality (RMI) proposed in Miller (1982) has become the standard testing tool in many RT studies. ${ }^{1}$ It stipulates that the RT distribution function for redundant stimuli is never larger than the sum of the RT distributions for the single stimuli. A violation of this inequality is interpreted as an indicator of an underlying neural summation (or coactivation) mechanism. When many different experimental conditions are to be compared, a numerical index of the amount of violation is very desirable. A widespread practice is to take a certain area with contours defined by the distribution functions for single and redundant stimuli. Here we show, for the first time, that this area can be interpreted in terms of mean RT differences, thus providing both a simple intuitive interpretation of the area and a means for statistical testing. ${ }^{2} \mathrm{We}$ also present a partial extension of this result to the trimodal stimulation condition.

We need the following notation. Let $R T_{X}$ and $R T_{Y}$ denote the processing time for the detection of signal $s_{X}$, and, respectively, $s_{Y}$, when presented alone, and let $R T_{X Y}$ denote the processing time when both signals $s_{X}$ and $s_{Y}$ are present. For simplicity, it is assumed here that detection latencies are identical to the observable RTs. Note that, because $R T_{X}, R T_{Y}$, and $R T_{X Y}$ are measured under different experimental conditions, there is no natural probability space to define their joint distribution. However, the race model assumptions can be stated explicitly using the "equal-indistribution" notion: Two random variables $U$ and $V$ are equal in distribution $\left(U={ }_{s t} V\right)$ when they have distribution functions of identical form. The race model assumes that (a) there is a nonnegative random vector $(X, Y)$ (defined by a distribution with respect to some probability space) such that $R T_{X Y}={ }_{s t} \min (X, Y)$, and (b) $X={ }_{s t} R T_{X}, Y={ }_{s t} R T_{Y}$. The latter assumption is often referred to as context invariance, stipulating that the signal detec-

\footnotetext{
${ }^{1}$ According to the Web of Science (May, 2005), Miller's (1982) article has 198 citations, with 60 of them over the last 3 years.

${ }^{2}$ Miller (1986) introduced the use of this geometric measure to assess the degree of violation of the inequality and also pioneered a bootstrapping test. For reasons unbeknown to us, the latter seems to have been ignored completely in the subsequent literature.
} 
tion latency distributions for $s_{X}$ and $s_{Y}$ are identical in single and redundant signal trials (Ashby \& Townsend, 1986; Luce, 1986).

With $E$ standing for the expected value of random variables, it follows, as a special case of Jensen's inequality (e.g., Billingsley, 1979), that

$$
E[\min (X, Y)] \leq \min [E(X), E(Y)]
$$

for any distribution of $(X, Y)$. Random variables $X$ and $Y$ are not observable (only their minimum is, in the redundant signal condition), but from the equal-in-distribution assumptions a testable analogue of Inequality 1 follows:

$$
E\left(R T_{X Y}\right) \leq \min \left[E\left(R T_{X}\right), E\left(R T_{Y}\right)\right]
$$

Testing the Race Model

The latter inequality has been used to test the race model on the level of average RTs. For example, in an RT stimulation, Gielen et al. (1983) obtained mean RTs for bimodal (visual-auditory and visualkinesthetic) and unimodal stimuli. In order to derive the race model's prediction of mean bimodal RT, they assumed stochastic independence between the two detection times in the bimodal condition and found average bimodal RTs to be smaller than predicted by the model, leading them to a rejection of the race model.

Note, however, that the validity of the inequality in Equation 2 is not restricted to the case of stochastic independence. This is important because dependent processing does affect the predictions of the race model. Indeed, assuming negative dependencethat is, relatively fast detection latencies for signal $s_{X}$ co-occur with relatively slow detection latencies for signal $s_{Y}$ and vice versa-it is obvious that the smaller of the two random latencies $R T_{X}$ and $R T_{Y}$ tends to be small as compared with the smaller of two independent latencies, as long as the individual latencies' means do not vary. ${ }^{3}$ The difficulty Gielen et al. (1983) faced was how to derive predictions of a dependent race model without restricting the model by specific distributional assumptions.

A more general test of the race model was developed by Miller $(1978,1982)$ in showing that

$$
P\left(R T_{X Y} \leq t\right) \leq P\left(R T_{X} \leq t\right)+P\left(R T_{Y} \leq t\right)
$$

must hold for all $t \geq 0$. This RMI follows from

$$
P[\min (X, Y) \leq t] \leq P(X \leq t)+P(Y \leq t)
$$

a special case of Boole's inequality (Billingsley, 1979). RMI and some of its variations and generalizations have been the subject of numerous theoretical and methodological studies (Ashby \& Townsend, 1986; Colonius, 1986, 1990, 1999; Colonius \& Ellermeier, 1997; Colonius \& Townsend, 1997; Colonius \& Vorberg, 1994; Diederich, 1992; Miller, 1986, 1991, 2004; Miller \& Ulrich, 2003; Mordkoff \& Yantis, 1991; Townsend \& Nozawa, 1995, 1997; Townsend \& Wenger, 2004; Ulrich \& Giray, 1986; Ulrich \& Miller, 1997).

Violations of RMI have been observed in many multimodal stimulation experiments but also under unimodal stimulation (e.g., Turatto, Mazza, Savazzi, \& Marzi, 2004). A common way to depict the amount of RMI violation is to subtract the single signal distributions from the redundant signals distribution

$$
P\left(R T_{X Y} \leq t\right)-P\left(R T_{X} \leq t\right)-P\left(R T_{Y} \leq t\right)
$$

and to plot this as a function $R^{*}{ }_{X Y}$, say, of $t$ (Miller, 1986):

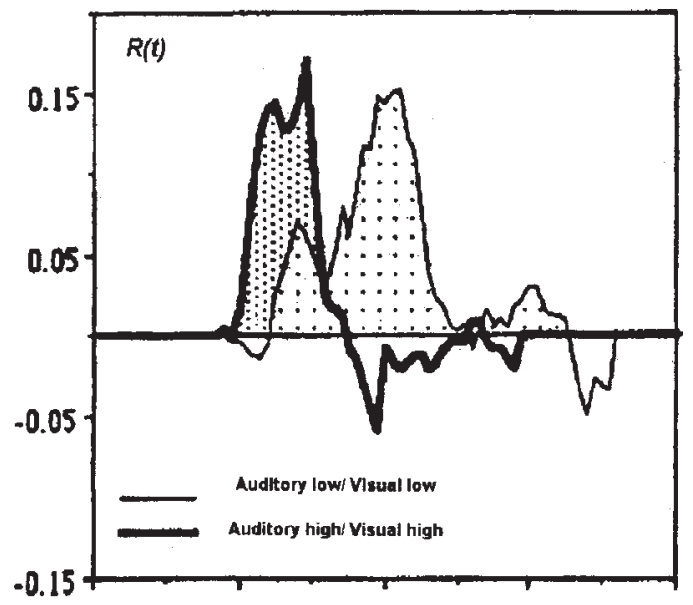

Figure 1. Areas above horizontal line represent the amount of violation of race model inequality for saccadic reaction times in two different stimulus conditions (after Nozawa et al., 1994). From "Parallel and serial processes in the human oculomotor system: Bimodal integration and express saccades," by G. Nozawa, P. A., Reuter-Lorenz, and H. C. Hughes, 1994, Biological Cybernetics, 72, 19-34. Copyright 1994 by SpringerVerlag. Adapted with permission.

$$
R_{X Y}^{*}(t)=P\left(R T_{X Y} \leq t\right)-P\left(R T_{X} \leq t\right)-P\left(R T_{Y} \leq t\right) .
$$

By the inequality shown in Equation 3, positive values of $R^{*}{ }_{X Y}$ $(t)$ indicate violations of RMI. For example, Figure 1 presents functions $R^{*}{ }_{X Y}(t)$ from two different stimulus conditions in a visual-auditory saccadic RT study by Nozawa, Reuter-Lorenz, and Hughes (1994).

Given that the left-hand side of the inequality shown in Equation 3 is always bounded by 1 , the inequality can be rewritten as

$$
P\left(R T_{X Y} \leq t\right) \leq \min \left[P\left(R T_{X} \leq t\right)+P\left(R T_{Y} \leq t\right), 1\right],
$$

resulting in a slightly modified function

$$
R_{X Y}(t)=P\left(R T_{X Y} \leq t\right)-\min \left[P\left(R T_{X} \leq t\right)+P\left(R T_{Y} \leq t\right), 1\right] .
$$

Violations of RMI will again result in positive values of $R T_{X Y}(t)$, whereas negative or zero values of $R T_{X Y}(t)$ are compatible with the race model. ${ }^{4}$

\section{Assessing the Amount of RMI Violation}

The amount of violation is typically interpreted as the strength of neural summation or coactivation, that is, the amount of response facilitation that is not reducible to probability summation (viz., statistical facilitation). If many different experimental conditions are to be compared with respect to their degree of RMI violation, reducing the information contained in $R T_{X Y}(t)$ or $R T^{*}{ }_{X Y}(t)$ to a single numerical index of neural summation is very desirable. It has become common practice to interpret the area

\footnotetext{
${ }^{3}$ Positive dependence has the opposite effect: In the extreme case of perfect positive dependence, the smaller of the two random latencies will have the same mean as the one with the smaller mean.

${ }^{4}$ Because there is some arbitrariness, slightly different definitions of $R_{X Y}(t)$ occur in the literature. The version chosen here is best suited for our purposes.
} 
under positive $R T_{X Y}(t)$ or $R T^{*}{ }_{X Y}(t)$ values $V^{+}$, say, as a quantitative measure of the amount of violation of RMI and, thereby, of neural summation. For example, in a study on summation, Hughes et al. (1998) plotted $V^{+}$values as a function of four different spatial positions of the auditory and three different positions of the visual stimulus (see Figure 2).

This geometric measure of RMI violation is simple and attractive, but it may seem a bit arbitrary. The following proposition, on the other hand, shows that a slight modification of this geometric measure has a deeper interpretation relating it directly to the aforementioned race model test on the level of average RTs.

Proposition 1: Let $E^{(-)}\left[\min \left(R T_{X}, R T_{Y}\right)\right]$ be the mean RT predicted by a race model with maximal negative dependence between the detection latencies $R T_{X}$ and $R T_{Y}$. Then the integral over function $R T_{X Y}(t)$

$$
V_{X Y}=\int_{0}^{\infty} R_{X Y}(t) d t=E^{(-)}\left[\min \left(R T_{X}, R T_{Y}\right)\right]-E\left(R T_{X Y}\right)
$$

where $E\left(R T_{X Y}\right)$ is the (observed) mean RT in the redundant signals condition.

This proposition is based on the fact that the right-hand side of the RMI in Equation 7,

$$
\min \left[P\left(R T_{X} \leq t\right)+P\left(R T_{Y} \leq t\right), 1\right],
$$

is a distribution function, namely, the distribution function of $\min \left(R T_{X}, R T_{Y}\right)$ with maximal negatively dependent $R T_{X}, R T_{Y}$ (for a proof, see Appendix A). A negative or zero value of $V_{X Y}$ indicates that the amount of observed RT facilitation is completely attainable by a race model, possibly with negative dependence

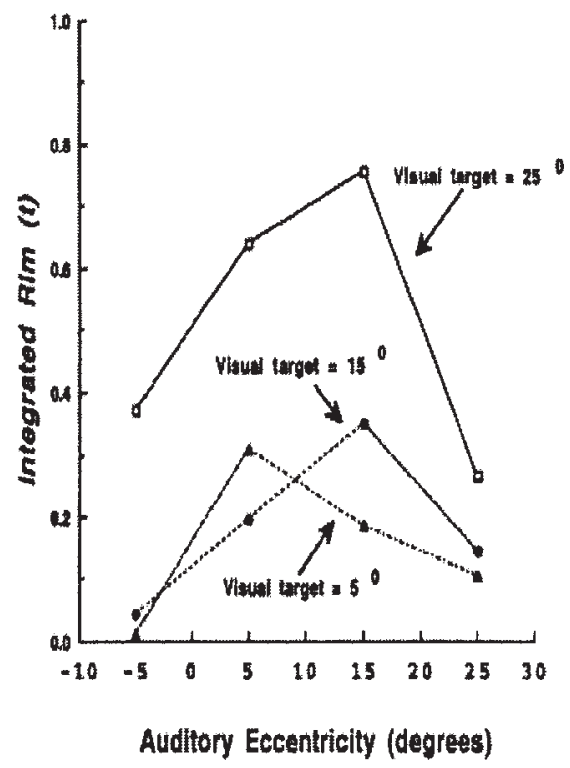

Figure 2. Positive areas under $R^{*}(t)$ as a function of visual and auditory stimulus position (after Hughes et al., 1998). From "Spatial characteristic of visual-auditory summation in human saccades," by H. C. Hughes, M. D. Nelson, and D. M. Aronchick, 1998, Vision Research, 38, 3955-3963. Copyright 1998 by Elsevier. Adapted with permission. between $R T_{X}$ and $R T_{Y}{ }^{5}$ The larger the positive values of $V_{X Y}$, the larger the amount of facilitation not explainable by the race model even if extreme negative dependence between the detection processing times is assumed.

Moreover, assuming that observable RTs include a variable base time, it can be shown that this implies a moderating effect on negative dependence leading to a possible underestimation of $V_{X Y}$ (see Colonius, 1990, Proposition 5.1) . ${ }^{6}$ In order to gauge the size of this underestimation, however, one would need to have an estimate of the base time variability, which may be difficult to obtain in practice.

Given that function $R_{X Y}(t)$ may be positive or negative depending on the value of $t$, index $V_{X Y}$ is equal to the area under $R_{X Y}(t)$ above the abscissa minus the area below the abscissa but above $R T_{X Y}(t)$. Numerical estimates of these areas can, in principle, be obtained through numerical integration. An attractive alternative, not requiring any area estimations, is using the method of antithetic variates (e.g., Thompson, 2000) to generate a pair of maximally negative dependent random variables from the two single signal distributions and to compute the mean of their minima (Colonius, 1990; Miller, 1986). We illustrate the aforementioned proposition and the numerical estimation of $E^{(-)}\left[\min \left(R T_{X}, R T_{Y}\right)\right]$ by a hypothetical visual-auditory interaction experiment.

\section{Example: Visual-Auditory Interaction in RT}

Using an artificial data set with known underlying RT distributions allows us to study the effect of different degrees of neural summation on the geometric index $V_{V A}$. For computational simplicity, we assume exponentially distributed visual and auditory processing times with intensity parameters $\lambda_{V}$ and $\lambda_{A}$, respectively, for the unimodal stimulus conditions. Bimodal processing time is also exponentially distributed, ${ }^{7}$ with parameter $\lambda_{V A}$. Obviously, for $\lambda_{V A}=\lambda_{V}+\lambda_{A}$, we have an independent race model, but for $\lambda_{V A}$ $>\lambda_{V}+\lambda_{A}$, violations of RMI occur, as illustrated by function $R_{V A}(t)$ 's being positive for a large range of $t$ values (see Figure 3).

Computation of area value $V_{V A}$ requires determination of the winner's mean in a race model with maximally negative dependence, $E^{(-)}\left[\min \left(R T_{V}, R T_{A}\right)\right]$, which—at the population level-is done by simple integration (cf. Appendix A). Each curve in Figure 3 corresponds to a different value of $\lambda_{V A}$, and area $V_{V A}$ increases monotonically with $\lambda_{V A}$, indicating an increasing amount of neural coactivation.

Numerical estimates of $E^{(-)}\left[\min \left(R T_{V}, R T_{A}\right)\right]$ from sample data are computed by the method of antithetic variates. Basically, the procedure is to take pairs of RT values from the single signal distributions as follows: Take the fastest RT from the $R T_{V}$ sample and the slowest RT from the $R T_{A}$ sample as the first pair, the next-to-the-fastest from the $R T_{V}$ sample and the next-to-theslowest from the $R T_{A}$ sample, and so on. For each pair, determine

\footnotetext{
${ }^{5}$ It should be noted, however, that nonviolation of RMI does not automatically validate a race model explanation. In fact, Ulrich and Miller (1997) developed a test that, in principle, may rule out race models even when RMI is not violated.

${ }^{6}$ We are grateful to two of the reviewers, Jim Townsend and Christopher Honey, for pointing this out.

${ }^{7}$ This model derives from the Marshall-Olkin bivariate exponential distribution, an important model in reliability theory (cf. Galambos \& Kotz, 1978).
} 


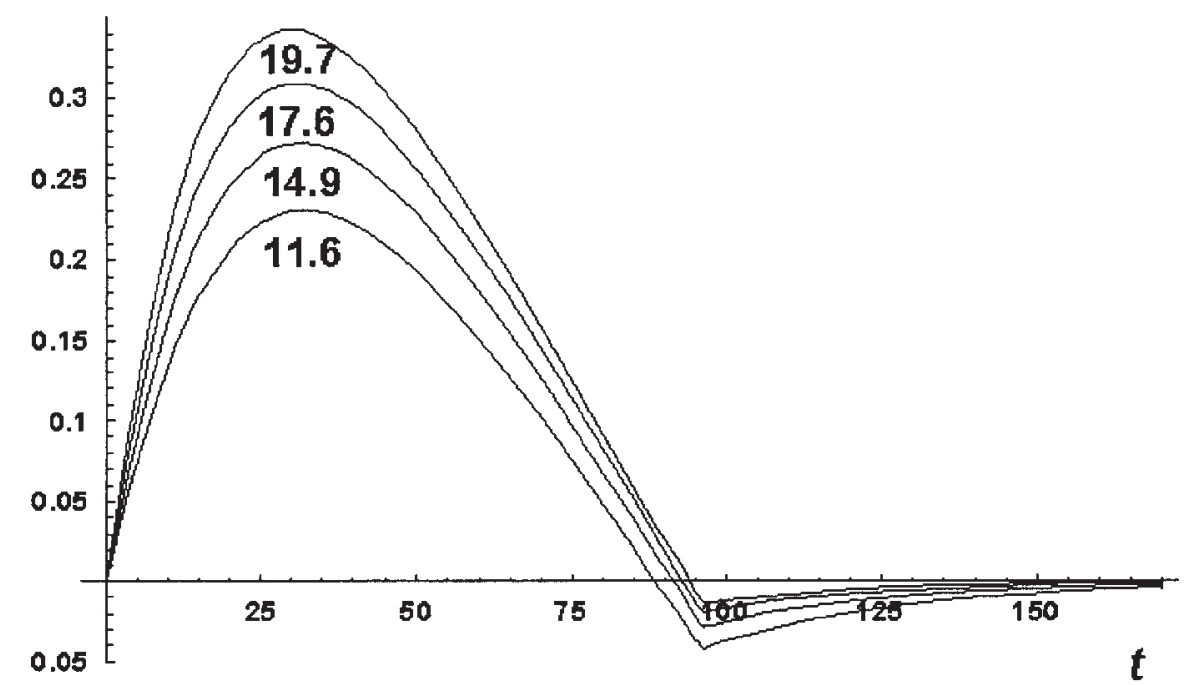

Figure 3. $\mathrm{R}_{V A}(t)$ functions of visual-auditory interaction example with $\lambda_{V}=.005, \lambda_{A}=.01$, and $\lambda_{V A}=.018$, $.022, .026$, and .03 , respectively. The corresponding $V_{V A}$ areas are indicated by the inscribed numbers.

the minimum and take the mean over these minima. Table 1 presents ordered samples of size $n=10$ from the $R T_{V}$ distribution $\left(\lambda_{V}=.005\right)$ and from the $R T_{A}$ distribution $\left(\lambda_{A}=.01\right)$.

The mean of the minima equals 59 as an estimate of $E^{(-)}\left[\min \left(R T_{V}, R T_{A}\right)\right]=42$. This estimate can, of course, be improved by taking larger, more realistic sample sizes.

\section{Statistical Testing of the Amount of RMI Violation}

In order to go beyond a descriptive measure of RMI violation, a statistical test for evaluating the null hypothesis of the inequality shown in Equation 3's being true is desirable. The index $V_{X Y}$, as a simple difference of independent means, is amenable to statistical testing of the null hypothesis of $V_{X Y} \leq 0$ by a conventional $t$ test (with nonhomogeneous variances) or a nonparametric (MannWhitney) $U$ test. $^{8}$ Another possible approach, already taken in Miller (1986), is to compute confidence intervals for the mean RT predicted by the (maximal negatively dependent) race model via bootstrapping from the observed single signal RT distributions (for details, see Miller, 1986).

Table 1

Ordered Samples of Size 10 From $R T_{v}$ and $R T_{A}$ Distributions (Columns 1 and 2) and Their Minima (Column 3)

\begin{tabular}{crr}
\hline Ordered $R T_{V}$ & Reverse-ordered $R T_{A}$ & Minima \\
\hline 68 & 349 & 68 \\
83 & 251 & 83 \\
86 & 156 & 86 \\
141 & 106 & 106 \\
147 & 90 & 90 \\
153 & 44 & 44 \\
154 & 42 & 42 \\
209 & 41 & 41 \\
380 & 22 & 22 \\
678 & 9 & 9 \\
\hline
\end{tabular}

Note. Average of the minima (third column) is 59.
Note that local violations of RMI may occur, although the corresponding $V_{X Y}$ value, as a global measure, may not show a significant violation. Thus, nonparametric tests at the level of the distribution functions will, in general, be more sensitive to violations of the race model. In this vein, Maris and Maris (2003) developed an interesting Kolmogorov-Smirnov-type test, but it is restricted to experimental paradigms where the single signal responses are drawn from a mixture distribution of the single signal distributions. No general solution in this direction is available yet.

\section{Extension to Three Redundant Signals}

The notion of a race easily extends to the case of more than two processes unfolding in time. A prominent example is the paradigm of multimodal stimulation with stimuli from the visual, auditory, and somatosensory modality (as early as Todd, 1912). Assuming (a) $R T_{X Y Z}={ }_{s t} \min (X, Y, Z)$; (b) $R T_{X Y}={ }_{s t} \min (X, Y), R T_{Y Z}={ }_{s t}$ $\min (Y, Z), R T_{X Z}={ }_{s t} \min (X, Z)$; and (c) $X={ }_{s t} R T_{X}, Y={ }_{s t} R T_{Y}$, and $Z={ }_{s t} R T_{Z}$, an extension of the inequality in Equation 2,

$$
E\left(R T_{X Y Z}\right) \leq \min \left[E\left(R T_{X}\right), E\left(R T_{Y}\right), E\left(R T_{Z}\right)\right],
$$

predicts statistical facilitation again. There is a dramatic difference, however, between the bivariate and the multivariate (greater than 2) situation as far as the role of statistical dependence is concerned. By an elementary observation, three random variables cannot be pairwise negatively dependent to an arbitrarily high degree. Thus, although the direct extension of RMI,

$$
\begin{aligned}
P\left(R T_{X Y Z} \leq t\right) \leq \min \left[P\left(R T_{X} \leq t\right)\right. & \\
& \left.+P\left(R T_{Y} \leq t\right)+P\left(R T_{Z} \leq t\right), 1\right],
\end{aligned}
$$

\footnotetext{
${ }^{8}$ Independence can be assumed by constructing a joint probability space from the three separate experimental conditions (single and redundant stimuli). The sample estimate for $E^{(-)}\left[\min \left(R T_{X}, R T_{Y}\right)\right]$ is a function of the order statistics of the two single stimulus conditions and is thus independent of the estimate for $E\left(R T_{X Y}\right)$ from the redundant stimuli condition.
} 
obviously holds, its right-hand side does not, in general, constitute a distribution function for $\min \left(R T_{X}, R T_{Y}, R T_{Z}\right)$ (cf. Joe, 1997), thus preventing a direct generalization of Proposition 1.

Nevertheless, alternative distribution inequalities exist that lend themselves to geometric interpretation. One example is (Diederich, $1992)^{9}$

$$
P\left(R T_{X Y Z} \leq t\right) \leq P\left(R T_{X Y} \leq t\right)+P\left(R T_{Y Z} \leq t\right)-P\left(R T_{Y} \leq t\right),
$$

which follows from

$$
\begin{aligned}
P[\min (X, Y, Z) \leq t] \leq P[ & \min (X, Y) \leq t] \\
& +P[\min (Y, Z) \leq t]-P(Y \leq t) .
\end{aligned}
$$

This inequality has recently been tested in a multimodal stimulation experiment in Diederich and Colonius (2004). Figure 4 presents an example from a trimodal condition where the area between the upper curve (observed trimodal RT) and the middle one (right-hand side of the inequality in Equation 13) suggests a violation of the inequality.

An analogue to Proposition 1 gives the following interpretation of this area.

Proposition 2 (for proof see Appendix B): The integral over function

$$
\begin{aligned}
R_{X Y Z}(t)=P( & \left.R T_{X Y Z} \leq t\right) \\
- & {\left[P\left(R T_{X Y} \leq t\right)+P\left(R T_{Y Z} \leq t\right)-P\left(R T_{Y} \leq t\right)\right] }
\end{aligned}
$$

equals

$$
\begin{aligned}
V_{X Y Z}=\int_{0}^{\infty} R_{X Y Z}(t) d t=[ & E\left(R T_{X Y}\right) \\
& \left.+E\left(R T_{Y Z}\right)-E\left(R T_{Y}\right)\right]-E\left(R T_{X Y Z}\right) .
\end{aligned}
$$

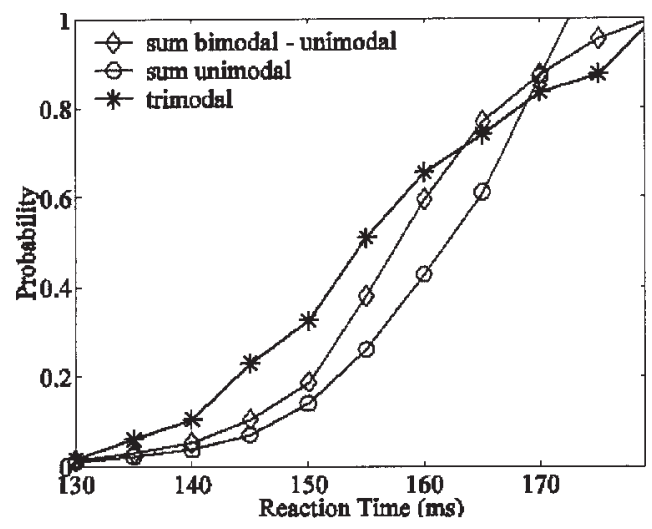

Figure 4. Trimodal stimulation result: Area between the upper and middle curves represents the value of $\mathrm{V}_{\mathrm{XYZ}}$ from Proposition 2. From "Bimodal and trimodal multisensory enhancement of reaction time: Effects of stimulus onset and intensity," by A. Diederich and H. Colonius, 2004, Perception \& Psychophysics, 66, 1388-1404. Copyright 2004 by the Psychonomic Society. Adapted with permission.
Thus, a positive value of $V_{X Y Z}$ indicates that mean RT with three signals is faster than predicted from the race model. It is interesting to note that Equation16 measures violation over and above that possibly caused with two signals. Indeed, let us assume that there are $V_{X Y}>0$ and $V_{Y Z}>0$ such that

$$
E\left(R T_{X Y}\right)=E^{(-)}\left[\min \left(R T_{X}, R T_{Y}\right)\right]-V_{X Y}
$$

and

$$
E\left(R T_{Y Z}\right)=E^{(-)}\left[\min \left(R T_{Y}, R T_{Z}\right)\right]-V_{Y Z}
$$

Inserting these expression into Equation 16 suggests that the RMI violations with two signals have already been discounted in the computation of $V_{X Y Z}$ :

$$
\begin{aligned}
V_{X Y Z}=\left\{E^{(-)}\left[\min \left(R T_{X}, R T_{Y}\right)\right]\right. & \left.-V_{X Y}\right\}+\left\{E^{(-)}\left[\min \left(R T_{Y}, R T_{Z}\right)\right]\right. \\
& \left.-V_{Y Z}\right\}-E\left(R T_{Y}\right)-E\left(R T_{X Y Z}\right) .
\end{aligned}
$$

\section{Conclusion}

We have shown that a commonly used geometric measure of the amount of violation of the race model relates performance to the case of maximal negative dependence between the two processing times and, specifically, that it equals a simple difference of mean RTs amenable to statistical testing. A direct generalization of this result to the processing of three or more signals was shown to be impossible in principle, but alternative geometric measures assessing race model violations, again expressible as mean RT differences, can be developed as demonstrated here for the trivariate situation.

${ }^{9}$ Two more inequalities of the same type follow from symmetry, with $X$, or $Z$, taking over the role of $Y$. Replacing the right-hand side of the inequality in Equation 13 by the minimum over all three possible upper bounds leads to a possibly sharper inequality generalizing the subsequent development. However, for ease of exposition, we abstain from presenting the more general case.

\section{References}

Amlôt, R., Walker, R., Driver, J., \& Spence, C. (2003). Multimodal visual-somatosensory integration in saccade generation. Neuropsychologia, 41, 1-15

Ashby, F. G., \& Townsend, J. T. (1986). Varieties of perceptual independence. Psychological Review, 93, 154-179.

Billingsley, P. (1979). Probability and measure. New York: Wiley.

Blake, R., Martens, W., \& DiGianfillipo, A. (1980). Reaction time as a measure of binocular interaction in human vision. Investigative Ophthalmology, 19, 930-941.

Colonius, H. (1986). Measuring channel dependence in separate activation models. Perception \& Psychophysics, 40, 251-255.

Colonius, H. (1990). Possibly dependent probability summation of reaction time. Journal of Mathematical Psychology, 34, 253-275.

Colonius, H. (1999). A theorem on parallel processing models with a generalized stopping rule. Mathematical Social Sciences, 38, 247-258.

Colonius, H., \& Ellermeier, W. (1997). Distribution inequalities for parallel models of reaction time with an application to auditory profile analysis. Journal of Mathematical Psychology, 41, 19-27.

Colonius, H., \& Townsend, J. T. (1997). Activation-state representation of models for the redundant signals effect. In A. A. J. Marley (Ed.), Choice, decision, and measurement: Essays in honor of R. Duncan Luce (pp. 245-254). Mahwah, NJ: Erlbaum. 
Colonius, H., \& Vorberg, D. (1994). Distribution inequalities for parallel models with unlimited capacity. Journal of Mathematical Psychology, $38,35-58$.

Corballis, M. C. (1998). Interhemispheric neural summation in the absence of the corpus callosum. Brain, 121, 1795-1807.

Diederich, A. (1992). Probability inequalities for testing separate activation models of divided attention. Perception \& Psychophysics, 52, 714-716.

Diederich, A. (1995). Intersensory facilitation of reaction time: Evaluation of counter and diffusion coactivation models. Journal of Mathematical Psychology, 39, 197-215.

Diederich, A., \& Colonius, H. (1987). Intersensory facilitation in the motor component ? A reaction time analysis. Psychological Research, 49, 23-29.

Diederich, A., \& Colonius, H. (2004). Bimodal and trimodal multisensory enhancement of reaction time: Effects of stimulus onset and intensity. Perception \& Psychophysics, 66, 1388-1404.

Diederich, A., Colonius, H., Bockhorst, D., \& Tabeling, S. (2003). Visualtactile interaction in saccade generation. Experimental Brain Research, 148, 328-337.

Galambos, J., \& Kotz, S. (1978). Characterizations of probability distributions. Berlin, Germany: Springer-Verlag.

Gielen, S. C. A. M., Schmidt, R. A., \& Van den Heuvel, P. Y. M. (1983). On the nature of intersensory facilitation of reaction time. Perception \& Psychophysics, 34, 161-168.

Hughes, H. C., Nelson, M. D., \& Aronchick, D. M. (1998). Spatial characteristic of visual-auditory summation in human saccades. Vision Research, 38, 3955-3963.

Joe, H. (1997). Multivariate models and dependence concepts. London: Chapman \& Hall.

Luce, R. D. (1986). Response times: Their role in inferring elementary mental organization. New York: Oxford University Press.

Maris, G., \& Maris, E. (2003). Testing the race model inequality: A nonparametric approach. Journal of Mathematical Psychology, 47, 507514.

Marzi, C. A., Smania, N., Martini, M. C., Gambina, G., Tomerelli, G., Palamara, A., et al. (1996). Implicit-redundant target effects in visual extinction. Neuropsychologia, 34, 9-22.

Miller, J. O. (1978). Multidimensional same-different judgments: Evidence against independent comparisons of dimensions. Journal of Experimental Psychology: Human Perception and Performance, 4, 411422.

Miller, J. O. (1982). Divided attention: Evidence for coactivation with redundant signals. Cognitive Psychology, 14, 247-279.

Miller, J. O. (1986). Timecourse of coactivation in bimodal divided attention. Perception \& Psychophysics, 40, 331-343.

Miller, J. O. (1991). Channel interaction and the redundant targets effect in bimodal divided attention. Journal of Experimental Psychology: Human Perception and Performance, 17, 160-169.

Miller, J. O. (2004). Exaggerated redundancy gain in the split brain: A hemispheric coactivation account. Cognitive Psychology, 49, 118-154.
Miller, J. O., \& Ulrich, R. (2003). Simple reaction time and statistical facilitation: A parallel gains model. Cognitive Psychology, 46, 101-151.

Molholm, S., Ritter, W., Javitt, D., \& Foxe, J. J. (2004). Multisensory visual-auditory object recognition in humans: A high-density electrical mapping study. Cerebral Cortex 14, 452-465.

Mordkoff, J. T., \& Yantis, S. (1991). An interactive race model of divided attention. Journal of Experimental Psychology: Human Perception and Performance, 17, 520-538.

Nozawa, G., Reuter-Lorenz, P. A., \& Hughes, H. C. (1994). Parallel and serial processes in the human oculomotor system: Bimodal integration and express saccades. Biological Cybernetics, 72, 19-34.

Raab, D. H. (1962). Statistical facilitation of simple reaction times. Transactions of the New York Academy of Sciences, 24, 574-590.

Reuter-Lorenz, P. A., Nozawa, G., Gazzaniga, M. S., \& Hughes, H. C. (1995). Fate of neglected targets: A chronometric analysis of redundant target effects in the bisected brain. Journal of Experimental Psychology: Human Perception and Performance, 21, 211-230.

Savazzi, S., \& Marzi, C. A. (2004). The superior colliculus subserves interhemispheric neural summation in both normals and patients with a total section or agenesis of the corpus callosum. Neuropsychologia, 42, $1608-1618$.

Schwarz, W., \& Ischebeck, A. (1994). Coactivation and statistical facilitation in the detection of lines. Perception, 23, 157-168.

Thompson, J. R. (2000). Simulation: A modeler's approach. New York: Wiley.

Todd, J. W. (1912). Reactions to multiple stimuli. In R. S. Woodworth (Ed.), Archives of psychology: No. 25. Columbia contributions to philosophy and psychology (Vol. 21, No. 8). New York: Science Press.

Townsend, J. T., \& Nozawa, G. (1995). Spatio-temporal properties of elementary perception: An investigation of parallel, serial, and coactive theories. Journal of Mathematical Psychology, 39, 321-359.

Townsend, J. T., \& Nozawa, G. (1997). Serial exhaustive models can violate the race model inequality: Implications for architecture and capacity. Psychological Review, 104, 595-602.

Townsend, J. T., \& Wenger, M. J. (2004). A theory of interactive parallel processing: New capacity measures and predictions for a response time inequality series. Psychological Review, 111, 1003-1035.

Turatto, M., Mazza, V., Savazzi, S., \& Marzi, C. A. (2004). The role of the magnocellular and parvocellular systems in the redundant target effect. Experimental Brain Research, 158, 141-150.

Ulrich, R., \& Giray, M. (1986). Separate-activation models with variable base time: Testability and checking of cross-channel dependency. Perception \& Psychophysics, 36, 545-558.

Ulrich, R., \& Miller, J. O. (1997). Tests of race models for reaction time experiments with asynchronous redundant targets. Journal of Mathematical Psychology, 41, 367-381.

Westendorf, D. H., \& Blake, R. (1988). Binocular reaction times to contrast increments. Vision Research, 28, 355-359. 


\section{Appendix A}

\section{Proof of Proposition 1}

Writing $F_{X Y}(t)=P\left(R T_{X Y} \leq t\right), F_{X}(t)=P\left(R T_{X} \leq t\right)$, and $F_{Y}(t)=P\left(R T_{Y}\right.$ $\leq t)$,

$$
\begin{aligned}
R_{X Y}(t) & =F_{X Y}(t)-\min \left[F_{X}(t)+F_{Y}(t), 1\right] \\
& =1-\min \left[F_{X}(t)+F_{Y}(t), 1\right]-\left[1-F_{X Y}(t)\right] \\
& =\max \left[1-F_{X}(t)-F_{Y}(t), 0\right]-\left[1-F_{X Y}(t)\right] .
\end{aligned}
$$

Integrating yields

$$
\begin{aligned}
\int_{0}^{\infty} R_{X Y}(t) d t & =\int_{0}^{\infty} \max \left[1-F_{X}(t)-F_{Y}(t), 0\right] d t \\
& -\int_{0}^{\infty}\left[1-F_{X Y}(t)\right] d t=E^{(-)}\left[\min \left(R T_{X}, R T_{Y}\right)\right]-E\left(R T_{X Y}\right),
\end{aligned}
$$

where $E^{(-)}$refers to the mean RT under maximally negative dependence between $R T_{X}$ and $R T_{Y}$ (cf. Colonius, 1990) and $E\left(R T_{X Y}\right)$ is the observed redundant signals mean $\mathrm{RT}$.

The last step follows from the equality

$$
\int_{0}^{\infty}\left[1-F_{X}(t)\right] d t=E(X)
$$

holding for any positive (continuous) random variable $X$ with distribution function $F_{X}$

\section{Appendix B}

Proof of Proposition 2

$$
\begin{aligned}
& \text { Writing } F_{X Y Z}(t)=P\left(R T_{X Y Z} \leq t\right) \text {, and so on, } \\
& \begin{array}{l}
R_{X Y Z}(t)=P\left(R T_{X Y Z} \leq t\right)-\left[P\left(R T_{X Y} \leq t\right)+P\left(R T_{Y Z} \leq t\right)-P\left(R T_{Y} \leq t\right)\right] \\
\quad=\left[1-F_{X Y}(t)\right]+\left[1-F_{Y Z}(\mathrm{t})\right]-\left[1-F_{Y}(t)\right]-\left[1-F_{X Y Z}(t)\right] . \quad(\mathrm{B} 1)
\end{array}
\end{aligned}
$$

Integrating yields

$$
\int_{0}^{\infty} R_{X Y Z}(t) d t=\left[E\left(R T_{X Y}\right)+E\left(R T_{Y Z}\right)-E\left(R T_{Y}\right)\right]-E\left(R T_{X Y Z}\right) .
$$

Received May 3, 2005

Revision received July 25, 2005

Accepted July 26, 2005 